\title{
Posterior Deltoid-to-Triceps Tendon Transfer for Elbow Extension in a Tetraplegia Patient: A Case Report
}

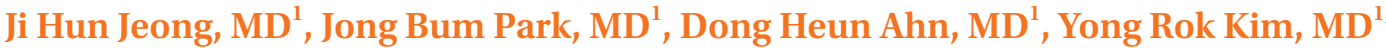 \\ Mi Jin Hong, $\mathrm{MD}^{1}$, Yung Jin Lee, $\mathrm{MD}^{1}$, Chang-il Park, $\mathrm{MD}^{1}$, Youn Moo Heo, $\mathrm{MD}^{2}$
}

Departments of ${ }^{1}$ Rehabilitation Medicine and ${ }^{2}$ Orthopedic Surgery, Konyang University College of Medicine, Daejeon, Korea

In tetraplegia patients, activities of daily living are highly dependent on the remaining upper limb functions. In other countries, upper limb reconstruction surgery to improve function has been applied to diverse cases, but few cases have been reported in Korea. The current authors experienced a case of posterior deltoid-to-triceps tendon transfer and rehabilitation in a complete spinal cord injury with a C6 neurologic level, and we introduce the case-a 36-year-old man-with a literature review. The patient's muscle strength in C5 C6 muscles were normal, but C7 muscles were trace, and the Spinal Cord Independence Measure III (SCIM III) score was 24. The tendon of the posterior deltoid was transferred to the triceps brachii muscle, and then the patient received comprehensive rehabilitative treatment. His $\mathrm{C} 7$ muscle strength in the right upper extremity was enhanced from trace to fair, and his SCIM III score improved to 29.

Keywords Tetraplegia, Tendon transfer, Upper extremity

\section{INTRODUCTION}

The incidence of tetraplegia caused by traumatic spinal cord injury (SCI) is decreasing, and advances in acutephase treatment after SCI and ventilator care have increased the survival rate as well as life expectancy [1]. In tetraplegia patients, activities of daily living (ADLs) are

Received March 20, 2015; Accepted July 13, 2015

Corresponding author: Jong Bum Park

Department of Rehabilitation Medicine, Konyang University College of Medicine, 158 Gwanjeodong-ro, Seo-gu, Daejeon 35365, Korea Tel: +82-42-600-6700, Fax: +82-42-600-9090, E-mail: jbocean@hanmail. net

(c) This is an open-access article distributed under the terms of the Creative Commons Attribution Non-Commercial License (http://creativecommons. org/licenses/by-nc/4.0) which permits unrestricted noncommercial use, distribution, and reproduction in any medium, provided the original work is properly cited.

Copyright (C) 2016 by Korean Academy of Rehabilitation Medicine largely influenced by the functions of the remaining upper limbs. Thus, for greater quality of life, it is very important to recover the functions of the upper limbs as much as possible. Studies on improving upper limb function after upper extremity reconstruction began to be reported from the 1970s. Beginning with Moberg's tenodesis of the flexor policis longus in 1975, there has been continuous improvement in surgery to substantially improve muscle function [2]. It was also reported that upper extremity reconstruction had a comprehensive positive effect on tetraplegia patients' ADLs, independence, quality of life, etc., and an 11-year-long follow-up study found that such benefits continued for many years [3-5]. In foreign countries, upper limb function surgery has been conducted, but in Korea, few cases have been reported, and there is also little experience with postoperative rehabilitation. 
The current authors experienced a case of a posterior deltoid-to-triceps tendon transfer and rehabilitation for elbow extension in a C6 tetraplegia patient, and we here report the case along with a literature review.

\section{CASE REPORT}

A 36-year-old man with complete C6 tetraplegia that occurred from comminuted fractures to the C5 and C6 caused by a car accident 11 years previously. After surgical treatment, he had been receiving rehabilitation therapy along with regular follow-up observation (Fig 1). The patient was highly motivated for relatively newer treatment options as evidenced by his active social life (wheelchair rugby, etc.), and our general understanding of the surgery and the patient's goals suggested that upper limb function surgery would improve the patient's ADL functioning and quality of life. On the manual muscle test (MMT), the C5 and C6 key muscles in the upper limbs were normal, but the $\mathrm{C} 7$ key muscles were trace. In muscle force measurement using hand-held dynamometers (PowerTrack AP1110; JTECH Medical, Midvale, UT, USA), the scores for the right $\mathrm{C} 5, \mathrm{C} 6$, and $\mathrm{C} 7$ were 125.5 $\mathrm{N}, 70.6 \mathrm{~N}$, and $0 \mathrm{~N}$, respectively (Tables 1,2 ). There was no passive limitation of motion and spasticity in the upper extremities. Proprioceptive sensation (Weber twopoint discrimination test) was intact. The patient's Spinal Cord Independence Measure III (SCIM III) score was 24,

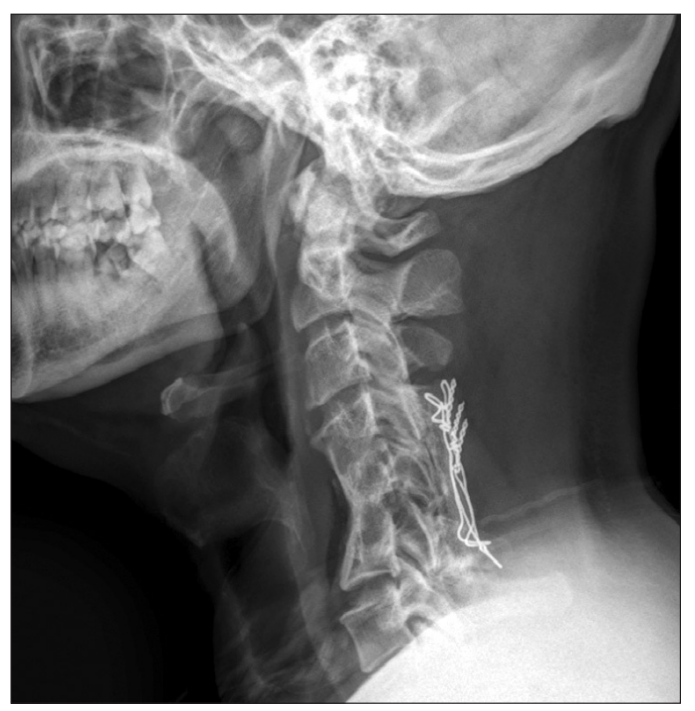

Fig. 1. Postoperative bone union status of comminuted fracture in the 5th and 6th cervical vertebrae. showing that he was dependent in self-care and mobility. A right posterior deltoid-to-triceps tendon transfer was performed in Konyang University Hospital (Fig. 2). The patient was casted for 5 weeks after the surgery, and from then orthosis that could adjust the angle of the elbow joint was applied by increasing the flexion angle of the elbow joint by $10^{\circ}$ per week. During the period with the orthosis, rehabilitation therapy was applied within limited range of motion (ROM), mainly for strengthening. Because the postoperative immobilization period had to be quite long, motor imagery therapy was initiated just after the surgery because it was relatively stable and the patient could experience and train on new functional uses of the transferred muscle. Motor imagery therapy was applied for the period of immobilization (10 weeks), 5 times per week, 30 minutes per session. Then, once an

Table 1. Motor grade and sensory level

\begin{tabular}{|lll}
\hline & Right & Left \\
\hline Motor grade & & \\
C5 & 5 & 5 \\
\hline C6 & 5 & 5 \\
C7 & 1 & 1 \\
C8 & 1 & 1 \\
T1 & 0 & 0 \\
\hline L2 & 0 & 0 \\
L3 & 0 & 0 \\
\hline L4 & 0 & 0 \\
\hline L5 & 0 & 0 \\
\hline S1 & 0 & 0 \\
\hline Sensory intact level & & \\
\hline Pain & C6 & C6 \\
Temperature & C6 & C6 \\
\hline Proprioception & T1 & T1 \\
\hline
\end{tabular}

Motor grade: 1 , trace; 2 , poor; 3 , fair; 4 , good; 5 , normal.

Table 2. Muscle force (N) in the right upper extremity

\begin{tabular}{lccc} 
& \multirow{2}{*}{ Preoperative } & \multicolumn{2}{c}{ Postoperative } \\
\cline { 3 - 4 } & & 11 wk & 24 wk \\
\hline Shoulder flexion & 114 & 89 & 126 \\
Shoulder extension & 87 & 54 & 86 \\
Shoulder abduction & 107 & 96 & 113 \\
\hline Elbow flexion & 125.5 & 100.2 & 147 \\
Elbow extension & 0 & 41.8 & 59 \\
\hline Wrist extension & 70.6 & 56 & 81.6 \\
\hline
\end{tabular}



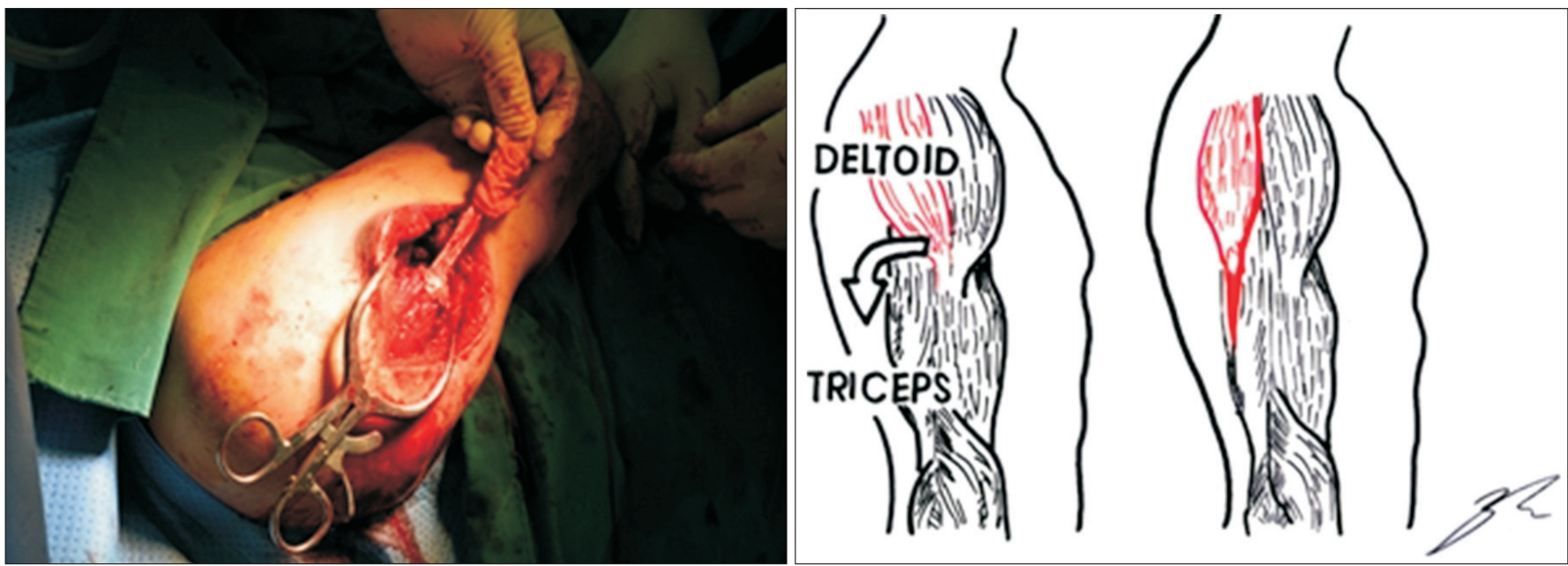

Fig. 2. The tendon transfer of the posterior deltoid to the triceps brachii muscle.

elbow brace was put on and the surgical site had been stabilized, biofeedback through surface electromyogram (EMG) therapy was applied 5 times per week, also 30 minutes per session. The patient could observe the EMG of his elbow extensors in real time on a video display screen and listen to audio feedback sound whose intensity was proportional to the strength of contraction. In the 11th week after the surgery, the patient had a followup evaluation. On the MMT, the C7 key muscle was improved from trace to fair, and with his muscle force, measured by hand-held dynamometers (PowerTrack), elbow extension increased from $0 \mathrm{~N}$ to $41.8 \mathrm{~N}$ (Table 2). His SCIM III score was 24 , the same as the first measurement. These results suggested that the muscle force of the elbow extensors was strengthened but was not associated with functional improvement. We applied comprehensive rehabilitative management continuously, and in the 24th week after the surgery, the patient was reevaluated. In the results, $\mathrm{C} 7$ key muscle motor function was fair, and muscle force measured with hand-held dynamometers (PowerTrack) improved to $59 \mathrm{~N}$. The patient's total SCIM III score increased to 29 based on functional improvements in ADLs such as upper body dressing, pressure relief, bed-to-wheelchair transfer, and wheelchair-totoilet/tub transfer (Table 3). Moreover, the muscular weakness in shoulder extension resulting from the transfer of the posterior deltoid was found to be insignificant. The muscle force of shoulder extension was $87 \mathrm{~N}$ before the surgery and $86 \mathrm{~N}$ in the 24 th week after, and the postoperative intensive rehabilitation therapy improved the
Table 3. SCIM III scores before and after the operation (24 weeks postoperative)

\begin{tabular}{lcc}
\hline & Preoperative & Postoperative \\
\hline $\begin{array}{l}\text { Dressing upper body } \\
\begin{array}{c}\text { Pressure relief } \\
\text { (4 activities) }\end{array}\end{array}$ & 2 & 1 \\
$\begin{array}{c}\text { Transfer - bed to } \\
\text { wheelchair }\end{array}$ & 0 & 4 \\
$\begin{array}{l}\text { Transfer - wheelchair } \\
\text { to toilet/tub }\end{array}$ & 0 & 1 \\
$\begin{array}{l}\text { Summation of } \\
\text { other items }\end{array}$ & 22 & 22 \\
Total SCIM III score & 24 & 29 \\
\hline
\end{tabular}

SCIM III, Spinal Cord Independence Measure III.

general muscle force in the upper limbs (Table 2).

\section{DISCUSSION}

The general objective of upper limb function surgery is to gain desired motion by fixing the tendon of a paralyzed muscle onto another, actively functioning, muscle on a proximal joint. Moberg [2] developed the method of posterior deltoid-to-triceps tendon transfer for active elbow extension and reported high patient satisfaction, improved muscle force, and stabilization of the upper limbs. According to Raczka et al. [6], 13 of 14 cases of posterior deltoid-to-triceps tendon transfer showed improvement in washing, personal hygiene, eating, compression- 
reducing motions for bedsore prevention, etc., and 2 of 4 cases that had the surgery on both sides showed significant improvement in their transfer activity. For the patient of our case as well, functional improvement was observed in ADLs such as upper body dressing, pressure relief, and transfer.

In performing tendon transfer, it is very important to establish the time of surgery and identify surgical indications. It is known to take approximately 6 months from injury to acquire neurologic stability, and tendon transfer is usually performed a year after the injury [7].

Preoperative tests should include a MMT, ROM, spasticity, proprioceptive sensation (Weber two-point discrimination test), and upper limb functional state, and it is important to include all of the muscles including the shoulder girdle in any motor function evaluation. Compared with MMTs, hand-held dynamometry can measure force quantitatively and, furthermore, it obtains various numeric data including peak force, endurance, power, and angle of maximal force.

Behavioral assessment is also important. The patient's motivation and present functional state should be assessed, and there should be discussion with the patient on what functional improvement can be practically expected from the surgery. Moreover, postoperative treatment plans for functional improvement should be explained sufficiently. Surgical indications need to be chosen in consideration of various factors including the patient's physical criteria, behavioral criteria, expected functional gains, and expectations and goals.

Postoperative rehabilitation follows a long-term protocol, and caution should be used against complications such as early lengthening of graft. In the usual process of rehabilitation therapy, 4-6 weeks' casting is followed by the application of an elbow brace that can adjust the angle of the elbow joint. For 6-8 weeks, the flexion angle of the elbow joint is increased by $10^{\circ}$ per week with the goal of full active extension under gravity. Until the 12th week after surgery, caution should be used against stretching the transferred muscle, and passive shoulder abduction should be limited to $45^{\circ}$ or less [8]. The most common complications are limited ROM and weakened muscle force caused by early graft lengthening [7]. For prevention of these complications, it is proposed to use a shoulder abduction brace to minimize complications.

In this case, the muscle force of the upper extremities measured in postoperative week 11 decreased considerably compared with the initial measurement, likely because of muscle wasting resulting from the 11 weeks' immobilization of the upper extremities; recovery and enhancement were attained through intensive rehabilitation therapy. For shoulder extension, it is believed that there must be the effect of the loss of muscle force caused by transferring the posterior deltoid muscle and tendon. However, muscle force measured in postoperative week 24 did not differ considerably from the initial measurement. This suggests that surgery by a skillful surgeon and adequate rehabilitation therapy may minimize the postoperative muscle weakness in shoulder extension caused by the loss of muscle force and immobilization.

The main objective of motor imagery therapy is to experience new functional uses and train on sensation for a transferred muscle. Due to the characteristics of tendon transfer, the period of postoperative immobilization is long, and there can be complications such as elongation, stretching, and tendon rupture during the period of rehabilitation therapy. Grangeon et al. [9] reported that tetraplegia patients who had tendon transfer received motor imagery therapy for elbow extension and showed improvement in muscle force and smoothness.

Wuolle et al. [4] reported high patient satisfaction after upper-extremity surgery for tetraplegia patients. In their survey of 67 participants (107 arms), most of them were satisfied with their surgery outcomes and were positively considering surgery for the opposite limb. Moreover, they reported that the treatment had positive effects on their lives including ADLs, independence, and occupational tasks.

In foreign countries, upper limb function surgery is being applied in various cases, and long-term benefits have been maintained. According to recent reports, the surgery has numerous advantages including the finding that tendon transfer patients' loss of muscle force resulting from aging was within the range of the normal population [5]; in Korea, however, such cases are rare. Lee et al. [10] reported on the state of upper extremity reconstruction for tetraplegia patients in Korea and on strategies for activating it. According to their survey on the current state of upper extremity reconstruction and clinicians' perceptions, only $2(6 \%)$ physiatrists and $6(21 \%)$ hand surgeons experienced upper extremity reconstruction clinically in person, and the main related factors were inexperience, 
lack of multidisciplinary cooperation, and lack of mutual referral.

One limitation of the current article is that the outcome of hand-held dynamometry can be affected by the measuring posture and the rater's physical condition. Therefore, it is difficult to set an absolute reference value. Furthermore, because inter-rater reliability is relatively poor, the evaluation is meaningful for a relative comparison between before and after treatment for the same subjects and by the same rater. Nevertheless, the use of hand-held dynamometry is advantageous over existing MMTs because it enables the acquisition of numeric data and can quantify muscle force.

We experienced a case of unilateral triceps tendon transfer and rehabilitation for elbow extension in a C6 tetraplegia patient, and here we report the case. More active research on upper limb function surgery as a part of aggressive rehabilitation therapy is expected to contribute to improving many tetraplegia patients' upper limb functions and quality of life.

\section{CONFLICT OF INTEREST}

No potential conflict of interest relevant to this article was reported.

\section{REFERENCES}

1. Bryce TN, Ragnarsson KT, Stein AB, Biering-Sorensen F. Spinal cord injury. In: Braddom RL, editor. Physical medicine and rehabilitation. 4th ed. Philadelphia: Saunders; 2011. p. 1293-346.

2. Moberg E. The present state of surgical rehabilita- tion of the upper limb in tetraplegia. Paraplegia 1987; 25:351-6.

3. Rogers JC, Figone JJ. Traumatic quadriplegia: followup study of self-care skills. Arch Phys Med Rehabil 1980;61:316-21.

4. Wuolle KS, Bryden AM, Peckham PH, Murray PK, Keith M. Satisfaction with upper-extremity surgery in individuals with tetraplegia. Arch Phys Med Rehabil 2003;84:1145-9.

5. Dunn JA, Rothwell AG, Mohammed KD, Sinnott KA. The effects of aging on upper limb tendon transfers in patients with tetraplegia. J Hand Surg Am 2014;39:31723.

6. Raczka R, Braun R, Waters RL. Posterior deltoid-totriceps transfer in quadriplegia. Clin Orthop Relat Res 1984;(187):163-7.

7. Friden J, Lieber RL. Evidence for muscle attachment at relatively long lengths in tendon transfer surgery. J Hand Surg Am 1998;23:105-10.

8. Bonds CW, James MA. Posterior deltoid-to-triceps tendon transfer to restore active elbow extension in patients with tetraplegia. Tech Hand Up Extrem Surg 2009;13:94-7.

9. Grangeon M, Guillot A, Sancho PO, Picot M, Revol $P$, Rode $G$, et al. Rehabilitation of the elbow extension with motor imagery in a patient with quadriplegia after tendon transfer. Arch Phys Med Rehabil 2010;91:1143-6.

10. Lee SY, Shin HI, Gong HS, Bin SW, Bang MS. Upper extremity reconstruction surgery for the persons with tetraplegia: current status and solutions to promote the procedures in Korea. J Korean Acad Rehabil Med 2008;32:175-81. 